\title{
Multiobjective Scheduling of Remote-Area Employees with Minimum Cost of Transportation
}

\author{
Hesham K. Alfares \\ Systems Engineering Department, King Fahd University of Petroleum \& Minerals, Dhahran 31261, Saudi Arabia \\ Correspondence should be addressed to Hesham K. Alfares; alfares@kfupm.edu.sa
}

Received 27 June 2014; Accepted 1 October 2014; Published 12 November 2014

Academic Editor: Fuh-Der Chou

Copyright (C) 2014 Hesham K. Alfares. This is an open access article distributed under the Creative Commons Attribution License, which permits unrestricted use, distribution, and reproduction in any medium, provided the original work is properly cited.

\begin{abstract}
An integer programming model and optimal solution procedure are presented for assigning employees to the $(10,14)$ days-off schedule. This schedule is used by a large oil company to schedule employees in remote work locations. The primary objective is to minimize the total number of employees. Since employees are flown to their remote work sites, the company also aims to minimize transportation cost. Therefore, secondary objectives include (1) minimizing the number of active days-off work patterns, (2) consistently using the same set of active days-off patterns, (3) assigning work schedules fairly among employees, and (4) avoiding the use of specialized optimization solvers. A rotation schedule is used in which two scheduling rules are enforced: a minimum proportion of weekend days off needs to be given and a maximum limit on the number of successive workdays cannot be exceeded. Utilizing the problem structure, simple optimal procedures are developed to solve this unique complex scheduling problem.
\end{abstract}

\section{Introduction}

This paper presents a mathematical programming model and an optimal solution methodology for a real-life daysoff scheduling problem. Specifically, the focus is on the (10, 14) days-off work schedule used for remote-area employees of a large oil company in the Middle East. The company's work operations include all aspects of the oil business, from exploration to producing, and cover a vast area of geographical expanse. Many permanent and temporary work sites such as drilling sites, oil wells, processing plants, pipelines, service stations, and oil platforms are located in remote areas both on-shore and off-shore. The company provides transportation to these remote areas by its own fleet of helicopters and small fixed-wing aircraft. The company's first objective is to reduce the labor cost by minimizing the workforce size in each work site. The second objective is to reduce the transportation cost by minimizing the total number of flights. In order to minimize the number of flight trips per week, the company applies certain work schedules for remote-area employees. Such schedules, including the (10, 14) schedule, are designed to have a lower frequency of daysoff breaks.
Under the $(10,14)$ days-off work schedule, each employee works for 10 consecutive days and takes a break of 4 consecutive off days during a 14-day work cycle. For each employee, this means one days-off break instead of two breaks in the two-week cycle and consequently half the number of flight trips. In order to avoid work stoppage, employees cannot take their four off days all at the same time. Any work interruption is not acceptable, as it may reduce revenue and undermine oil supply to the market. In order to keep the work running continuously, the four-day break times may be different for different employees. It should be noted that there is a roundtrip flight for each break time (start and end day), regardless of the number of employees. This makes it important to minimize the number of different break times, that is, the number of days-off work patterns that are active (assigned to employees). In order to reduce the number of flights further, employees that have the same break times can be pooled together from different (nearby) work sites. To allow such pooling, the same set of active days-off patterns (same break times) should be selected as consistently as possible, although labor demands may vary from one work site to another.

From the company's point of view, the $(10,14)$ days-off schedule has the advantage of reducing the number of flights 
to remote work locations. From the employees' perspectives, however, the $(10,14)$ schedule keeps them away longer from their families. Moreover, this schedule can cause employees to miss weekends with their families because their breaks may not always coincide with weekends. Employees obviously prefer to have some of their days-off breaks on weekends, when their families are home all day. Therefore, for the sake of keeping up employee morale, each employee is assured at least $50 \%$ of weekend days off.

Naturally, some days-off patterns (breaks) are going to be more desirable than others. Therefore, for fairness, employees should take turns (rotations) on all active (assigned) daysoff patterns. In going through the rotations, with each new two-week cycle, employees may switch from one day-off pattern to another. This makes it possible to have more than 10 consecutive workdays straddling two cycles. To maintain employee morale and productivity, the maximum work stretch (continuous work period) is not allowed to exceed 14 days. Most of the remote-area employees and supervisors concerned with the $(10,14)$ schedule are technicians who are not familiar with optimization tools. Therefore, the company would like a simple scheduling tool that eliminates the need to use specialized optimization software.

This paper presents an integer programming (IP) model of the above-described $(10,14)$ days-off scheduling problem, as well as an optimal solution methodology. Details of the problem, the model, and the solution procedure are given in subsequent sections according to the following order. First, recent relevant days-off scheduling literature is surveyed in Section 2. The IP model of the multiobjective $(10,14)$ days-off scheduling problem is formulated in Section 3. The minimum workforce size to satisfy all objectives and constraints is determined in Section 4. The optimal procedure for determining days-off assignments to satisfy different labor demands is described in Section 5. A real-life application and an example are presented in Section 6. Finally, conclusions and suggestions are given in Section 7.

\section{Literature Review}

Employee scheduling is a challenging optimization problem that has major implications on workforce cost, size, productivity, and morale. Therefore, employee scheduling is a well-studied applied optimization problem. The problem is generally classified into three types: shift (time-of-day) scheduling, days-off (days-of-week) scheduling, and tour (time-of-day and days-of week) scheduling. Alfares [1] and Ernst et al. [2] provide comprehensive reviews of literature on employee scheduling. Brunner [3] gives a more recent but specialized review, focusing on the scheduling of physicians in hospitals. This section is mostly concerned with employee day-off scheduling literature published since 2005.

Although the days-off scheduling problem is NP-complete according to Brucker et al. [4], mathematical properties of particular problem versions are utilized to develop efficient solution algorithms. Jarray [5] applies discrete tomography principles to develop a three-step, polynomial-time days-off scheduling algorithm. First, a set of linear equations is solved, then a binary matrix is constructed, and finally a greedy procedure is used. Costa et al. [6] apply another polynomialtime algorithm to determine optimum days-off schedules to satisfy variable daily labor demands. The days-off schedule for each employee is subject to several conditions: fixed number of workdays, two or three consecutive off days per week, and at least three consecutive off days per month. Jarray [7] develops a three-step decomposition algorithm to solve a compressed workweek (3-4 workdays) version of the problem that also includes weekend-off frequency constraints.

There are several applications of days-off scheduling in the service sector, especially in maintenance. Lilly et al. [8] develop a 4-day workweek schedule for maintenance employees at a power plant in Nigeria. They estimate that $10 \%$ savings in labor cost could be obtained by switching from five to four workdays per week for maintenance crews. Alfares [9] constructs a simulation model to evaluate alternative days-off schedules for a multi-craft pipeline maintenance workforce. Assuming stochastic daily demands for each craft, the model suggests a days-off schedule to reduce average completion times of maintenance orders. Considering limits on the number of workdays, weekends work, and maximum work stretch, Elshafei and Alfares [10] apply dynamic programming (DP) to determine optimal days-off assignments. The DP model assumes daily labor demands to be variable and costs of days-off patterns to be dependent on the given sequence of work and off days.

Transportation is a major service sector with several applications of days-off scheduling algorithms. Klinkert [11] uses an IP model with soft and hard constraints to construct days-off schedules for multiskilled airport ground handling employees. To facilitate solution, soft constraints are incorporated in the objective function, while a preprocessing IP model is used to identify the most critical hard constraints. Maenhout and Vanhoucke [12] address the airline crewscheduling problem, in which crew members are assigned to flight duties in order to satisfy a variable workload. Several approaches are presented for assigning airline crews to fixed days-off patterns, including cyclical schedules and ad hoc schedules. Kyngäs and Nurmi [13] apply a modified version of the cooperative local search method to generate optimal daysoff schedules for employees in a Finnish bus transportation company.

Air transportation applications of employee scheduling is an active area of research. Addressing a problem similar to Klinkert [11], Lusby et al. [14] formulate a cutting stock-based IP model for days-off scheduling of ground crews of an airline company. Assuming a schedule of six work days followed by three off days per cycle, decomposition and column-generation heuristics are used to develop efficient six-month employee schedules. Abdoul Soukour et al. [15] decompose airport security staff scheduling into three stages: days-off scheduling, shift scheduling, and staff assignment. A memetic algorithm is integrated with an evolutionary algorithm and local search techniques to solve this staff assignment problem.

By definition, employee tour scheduling involves a daysoff scheduling component. A number of tour scheduling algorithms have noteworthy and distinctive days-off scheduling features. Using implicit programming techniques, Rong [16] develops monthly tour schedules for a mixed workforce 
using both binary and general integer programming models. The unique feature of Rong's models is the inclusion of weekend off frequency constraints to maintain employees' morale. Assuming random labor demands for each department, Campbell [17] presents a two-stage stochastic IP approach for tour scheduling of cross-trained employees. In the first stage, days-off scheduling is used to allocate workdays, while in the second stage, shift scheduling is used to allocate work hours.

\section{Model Formulation}

As stated in Section 1, the company's main objective is to minimize the workforce size. The secondary objectives are (1) minimizing the number of active days-off work patterns, (2) assigning the same set of days-off patterns, (3) allocating work assignments fairly among employees, and (4) avoiding the need to use specialized optimization solvers. The objective function of the IP model, formulated below, explicitly includes only the minimum workforce size and the minimum number of days-off work patterns. The remaining secondary objectives are implicitly addressed in the overall solution process.

3.1. Assumptions. The optimization algorithm presented in this paper applies to a single-shift, 14-day-cycle days-off schedule according to the following conditions.

(1) Each employee is assigned 10 consecutive workdays and 4 consecutive off days per two-week cycle.

(2) The maximum work stretch is 14 consecutive workdays.

(3) During the rotation period, at least $50 \%$ of the daysoff breaks include weekends.

(4) The first objective is to minimize the total workforce size, and the second objective is to minimize the number of active days-off patterns.

(5) The demand for employees has one constant level for all five regular workdays and another constant level for the two weekend days.

(6) A cyclic rotation schedule is used, in which all employees take turns on the assigned days-off patterns.

\subsection{Model Variables and Parameters}

3.2.1. Decision Variables. $x_{j}=$ number of employees assigned to days-off work pattern $j, j=1,2, \ldots, 14$,

$$
\begin{gathered}
Q_{j}= \begin{cases}1, & \text { if } x_{j} \geq 1 \\
0, & \text { if } x_{j}=0\end{cases} \\
j=1,2, \ldots, 14
\end{gathered}
$$

$W=$ workforce size, that is, total number of assigned employees.

\subsubsection{Given Parameters.}

$a_{i j}= \begin{cases}1, & \text { if day } i \text { is a workday for days-off pattern } j \\ 0, & \text { otherwise }\end{cases}$

$$
i=1,2, \ldots, 14, \quad j=1,2, \ldots, 14
$$

$i=1, \ldots, 5$ and $i=8, \ldots, 12$ correspond to weekdays, $i=6,7,13,14$ correspond to the weekend, and $i=15$ corresponds to weekend-off frequency constraint (8).

Table 1 shows matrix $A=\left\{a_{i j}, i=1, \ldots, 15, j=1, \ldots, 14\right\}$, $\varepsilon=$ small positive constant $(0<\varepsilon \ll 1), D=$ number of workers required on each weekday, $E=$ number of workers required on each weekend day $(E \leq D), \rho=$ actual proportion of weekend-containing days off, that is, left-hand side of (8), $M=$ a large number, $M \geq W, J_{k}=$ set of days-off patterns with $k$ weekend days off per cycle, and $k=0,1,2$,

$$
\begin{aligned}
& J_{0}=\{1,2,8,9\} \\
& J_{1}=\{3,7,10,14\} \\
& J_{2}=\{4,5,6,11,12,13\} .
\end{aligned}
$$

3.3. The Integer Programming Model. According to assumptions stated above, the integer programming model of the (10, 14) days-off scheduling problem is shown as follows:

$$
\begin{array}{ll}
\text { Minimize } & Z=\sum_{j=1}^{14} x_{j}+\varepsilon \sum_{j=1}^{14} Q_{j} \\
\text { Subject to } \quad & \sum_{j=1}^{14} a_{i j} x_{j} \geq D, \quad i=1, \ldots, 5, i=8, \ldots, 12 \\
& \sum_{j=1}^{14} a_{i j} x_{j} \geq E, \quad i=6,7, i=13,14 \\
& x_{j} \leq M Q_{j} \quad j=1, \ldots, 14 \\
& 0.5 \sum_{j \in J_{1}} x_{j}+\sum_{j \in J_{2}} x_{j} \\
& \sum_{j=1}^{14} x_{j} \\
& \sum_{k=1}^{j+4} Q_{k}+\sum_{k=12}^{14} Q_{k} \geq Q_{j} \quad j=1, \ldots, 6 \\
& x_{j} \geq 0 \text { and integer, } j=1, \ldots, 14 \\
& Q_{j}=0 \text { or } 1, \quad j=1, \ldots, 14
\end{array}
$$

The primary objective in (4) is to minimize workforce size, which is the total number of employees $W$, while the secondary objective is to minimize the total number of active days-off patterns. Constraints (5) and (6) guarantee assigning at least the minimum number of required employees on workdays and weekends, respectively. Logical constraints (7) ensure that the binary variable $Q_{j}$ is equal to 1 only if days-off 
TABLE 1: Days-off matrix $A=\left\{a_{i j}\right\}$ for the 14 days-off work patterns.

\begin{tabular}{|c|c|c|c|c|c|c|c|c|c|c|c|c|c|c|}
\hline$i \backslash j$ & 1 & 2 & 3 & 4 & 5 & 6 & 7 & 8 & 9 & 10 & 11 & 12 & 13 & 14 \\
\hline 1 & 0 & 1 & 1 & 1 & 1 & 1 & 1 & 1 & 1 & 1 & 1 & 0 & 0 & 0 \\
\hline 2 & 0 & 0 & 1 & 1 & 1 & 1 & 1 & 1 & 1 & 1 & 1 & 1 & 0 & 0 \\
\hline 3 & 0 & 0 & 0 & 1 & 1 & 1 & 1 & 1 & 1 & 1 & 1 & 1 & 1 & 0 \\
\hline 4 & 0 & 0 & 0 & 0 & 1 & 1 & 1 & 1 & 1 & 1 & 1 & 1 & 1 & 1 \\
\hline 5 & 1 & 0 & 0 & 0 & 0 & 1 & 1 & 1 & 1 & 1 & 1 & 1 & 1 & 1 \\
\hline 6 & 1 & 1 & 0 & 0 & 0 & 0 & 1 & 1 & 1 & 1 & 1 & 1 & 1 & 1 \\
\hline 7 & 1 & 1 & 1 & 0 & 0 & 0 & 0 & 1 & 1 & 1 & 1 & 1 & 1 & 1 \\
\hline 8 & 1 & 1 & 1 & 1 & 0 & 0 & 0 & 0 & 1 & 1 & 1 & 1 & 1 & 1 \\
\hline 9 & 1 & 1 & 1 & 1 & 1 & 0 & 0 & 0 & 0 & 1 & 1 & 1 & 1 & 1 \\
\hline 10 & 1 & 1 & 1 & 1 & 1 & 1 & 0 & 0 & 0 & 0 & 1 & 1 & 1 & 1 \\
\hline 11 & 1 & 1 & 1 & 1 & 1 & 1 & 1 & 0 & 0 & 0 & 0 & 1 & 1 & 1 \\
\hline 12 & 1 & 1 & 1 & 1 & 1 & 1 & 1 & 1 & 0 & 0 & 0 & 0 & 1 & 1 \\
\hline 13 & 1 & 1 & 1 & 1 & 1 & 1 & 1 & 1 & 1 & 0 & 0 & 0 & 0 & 1 \\
\hline 14 & 1 & 1 & 1 & 1 & 1 & 1 & 1 & 1 & 1 & 1 & 0 & 0 & 0 & 0 \\
\hline 15 & -1 & -1 & 0 & 1 & 1 & 1 & 0 & -1 & -1 & 0 & 1 & 1 & 1 & 0 \\
\hline
\end{tabular}

pattern $j$ is active, that is, pattern $j$ is assigned to employees $\left(x_{j} \geq 1\right)$, and $Q_{j}$ is equal to 0 if pattern $j$ is not active, $\left(x_{j}=0\right)$.

The constraint on weekend work frequency (8) ensures that at least $50 \%$ of the days-off assignments contain weekends off. Numerator coefficients in constraint (8) indicate that patterns from the set $J_{1}$ contain half a weekend off, while patterns from the set $J_{2}$ contain a full weekend off. Constraint (8) can be alternatively expressed as follows:

$$
-\sum_{j \in J_{0}} x_{j}+\sum_{j \in J_{2}} x_{j} \geq 0 .
$$

Thus

$$
\begin{aligned}
& a_{15, j}=-1 \quad \text { if } j \in J_{0}, \\
& a_{15, j}=0 \quad \text { if } j \in J_{1}, \\
& a_{15, j}=1 \quad \text { if } j \in J_{2} .
\end{aligned}
$$

Constraints (9) enforce the maximum limit on work stretch length. In order for the work stretch not to exceed 14 consecutive days, some days-off patterns can be immediately followed only by certain subsets of patterns. Days-off pattern $j$, off on days $(j, j+1, j+2, j+3)$, can be followed only by patterns $(1, \ldots, j+4)$ and patterns $(12,13,14)$. Based on this rule, patterns $(7, \ldots, 14)$ can be followed by any pattern. Therefore, the work stretch constraints (9) are applied only to patterns $1, \ldots, 6$. Constraints (9) make sure that if pattern $j$ is assigned $\left(Q_{j}=1\right)$, then at least one of the feasibly-following patterns must also be assigned.

\section{The Minimum Workforce Size}

4.1. Bounds on Workforce Size W. Since the primary objective is to minimize workforce size $W$, it is necessary to initially focus on this objective and temporarily ignore the secondary objective. Therefore, at this stage, the objective function (4) is replaced by

$$
\text { Minimize } W=\sum_{j=1}^{14} x_{j} \text {. }
$$

The removal of the secondary objective entails the removal of the associated binary variables $Q_{1}, \ldots, Q_{14}$ and all related constraints. Therefore, the reduced IP model contains objective (14) subject only to labor demand constraints (5) and (6) weekends-off frequency constraints (8). Next, the dual model of this reduced primal model is formulated. Subsequently, using the procedure of Alfares [18], dual solutions are cyclically enumerated, and the dominant dual solutions are identified. The dominant dual solutions identified from the simplified $(10,14)$ model are associated with the following lower bounds on the workforce size $W$.

(1) First, the workforce size must be greater than the labor demand on any given day; thus

$$
W \geq \max (D, E) .
$$

(2) Since each employee is assigned 10 workdays per twoweek cycle, the total person-days assigned are equal to $10 \sum x_{j}=10 \mathrm{~W}$. This assignment must be greater than the total person-days required for two weeks, which is equal to $10 D+4 E$; thus

$$
W \geq D+0.4 E
$$

(3) The third bound can be obtained in several different ways. This bound is obtained by adding four daily labor demand constraints from (5) and (6). There are many four-day combinations (sets) that can be used to obtain this bound. When the four constraints in each set are added up, they give exactly $3 \sum x_{j}=3 W$ on 
the left-hand side and the sum of four daily demands on the right-hand side. For any $i(i=1, \ldots, 14)$, we define circular sets denoted by $T_{i}=\{i, i+4, i+7, i+11\}$. Since the $(10,14)$ schedule has a 14 -day cycle, $T_{i}$ are all mod 14 cyclic sets. Referring to matrix $A$ in Table 1 , each $x_{j}$ variable has at most three nonzero coefficients in the four rows of each set. For example, the sum of $T_{1}$ rows $(1,5,8$, and 12) from constraints (5) gives $3 \sum x_{j} \geq 4 D$, while the sum of $T_{2}$ rows $(2,6,9$, and 13) from constraints (5) and (6) gives $3 \sum x_{j} \geq 2 D+2 E$. Therefore

$$
W \geq \max \left(\frac{4 D}{3}, \frac{2 D+2 E}{3}\right) .
$$

(4) The fourth bound has to do with satisfying both weekend labor demands and weekends-off frequency requirements. Adding up weekend labor demand constraints (rows 6, 7, 13, and 14) and weekend off constraint (row 15) of Table 1 , we obtain $3 \sum x_{j} \geq 4 E$. Therefore

$$
W \geq \frac{4 E}{3}
$$

It is clear that the bound (15) is dominated by bounds (16) and (18). Choosing the highest of all applicable bounds and rounding up to the nearest integer value, the minimum workforce size $W$ is obtained as follows

$$
W=\left\lceil\max \left\{\frac{4 D}{3}, D+0.4 E, \frac{2 D+2 E}{3}, \frac{4 E}{3}\right\}\right\rceil,
$$

where $\lceil c\rceil=c$ rounded $u p$ to the nearest integer.

At this point, it should be noted that, in all remote work locations where the company applies the $(10,14)$ schedule, weekend labor demand never exceeds workdays labor demand; thus $E \leq D$. Dividing the arguments in (19) by $D$ and plotting them as functions of $B=E / D$, we obtain Figure 1. From the figure, it is clear that the last two functions in (19) are always dominated and hence not applicable for $B \leq 1(E \leq D)$. Therefore, for $E \leq D$, the minimum workforce size is given by

$$
W=\left\lceil\max \left\{\frac{4 D}{3}, D+0.4 E\right\}\right\rceil .
$$

4.2. Effect of Other Objectives and Constraints on $W$. Before proceeding further, it is essential to verify whether adding the remaining objectives and constraints can affect the minimum workforce size $W$ specified by (20). From the discussion on the different bounds on $W$, especially (17), it is clear that the integer programming model of the $(10,14)$ days-off scheduling problem is expected to have many alternative optimum solutions. Taking advantage of the existence of alternative optimum solutions, it is possible to select the optimum (minimum- $W$ ) solutions with the following features: (1) the minimum number of assigned days-off patterns, (2) similar sets of active days-off patterns for satisfying different labor demands $D$ and $E$, and (3) feasible work stretch lengths. Work

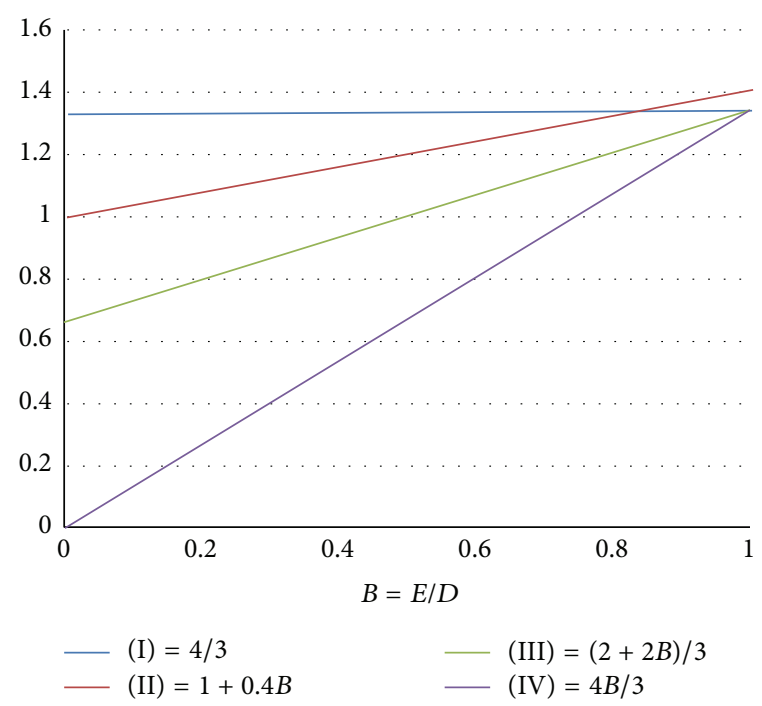

FIgURE 1: Workforce functions in (19), divided by $D$, versus $B=$ $E / D$.

stretch constraints are satisfied as long as a feasibly-following days-off pattern (such as 12,13, and 14) is active. Since some of the alternative solutions always contain these patterns, workstretch constraints do not affect workforce size $W$.

4.3. Rotation Scheme for the $W$ Employees. A cyclic rotation scheme is used to make sure weekend-off and work-stretch constraints are not violated and that the workload is equally distributed. Let us assume the workforce size $W$ and the number of employees assigned to each days-off pattern $\left(x_{1}, \ldots, x_{14}\right)$ are given. A feasible cyclic sequence of days-off assignments is then determined to guarantee the work stretch constrains are satisfied. This cyclic sequence determines the order in which days-off pattern $j$ is assigned. Since each days-off assignment lasts for 2 weeks, each employee will be assigned days-off pattern $j$ for $2 x_{j}$ weeks. Therefore, the length of the rotation cycle for all employees is $2 W$ weeks. During this cycle, all employees follow the same cyclic sequence but start in different weeks. Employee $w$ starts the sequence on week $S_{w}$, which is given by

$$
S_{w}=2 w-1, \quad w=1, \ldots, W .
$$

\section{Assigning Employees to Days-Off Patterns}

Given the values of labor demand levels for weekdays and weekends, $D$ and $E$, first the workforce size $W$ is calculated by (20). According to the applicable function in (20), primarydual complementary slackness rules are applied on the dual solution to identify basic variables and binding constrains in the corresponding primal model. Taking advantage of the high degeneracy of the $(10,14)$ problem, selection of the alternative primal solutions is directed towards assigning the same minimum set of active days-off patterns while satisfying work stretch constraints. Based on extensive numerical experimentation with different values of $D$ and $E$, five distinct 
solutions are identified. These five solutions, in which the number of active days-off patterns is equal to $4,7,8,10$, or 11, are presented below.

5.1. Four Active Patterns. This solution applies when $W=$ $\lceil 4 D / 3\rceil$ under certain conditions to be discussed below. Given that $W=\lceil 4 D / 3\rceil$, we can select a minimum of four active days-off patterns $(4,6,10,14)$. On each weekday, three out of these four patterns are working. In the first weekend (days 6 and 7), however, only patterns 10 and 14 are working. Assuming $\left(x_{4}=x_{6}\right)$ and $\left(x_{10}=x_{14}\right)$ and ignoring dominated constraints, the applicable labor demand constraints for weekdays (5) and weekends (6) can be expressed as

$$
\begin{gathered}
x_{4}+2 x_{10} \geq D \\
2 x_{4}+x_{10} \geq D \\
2 x_{10} \geq E,
\end{gathered}
$$

where

$$
2 x_{4}+2 x_{10}=W .
$$

Combining (22b) with (22d) gives

$$
x_{4} \geq D-0.5 W \text {. }
$$

Combining (22d) with (22a) gives

$$
x_{4} \leq W-D \text {. }
$$

Combining (22d) with (22c) gives

$$
x_{4} \leq 0.5 W-0.5 E \text {. }
$$

A feasible four-pattern solution exists if the lower bound in (23a) is not greater than the upper bounds in (23b) and (23c). Considering (23a) with the upper bound in (23b) and setting

$$
D-0.5 W \leq W-D
$$

we obtain

$$
\frac{4 D}{3} \leq W
$$

This is redundant since $W=\lceil 4 D / 3\rceil$. Therefore, $(23 \mathrm{~b})$ is redundant and can be ignored.

Considering (23a) with the upper bound in (23c) and setting

$$
D-0.5 W \leq 0.5 W-0.5 E,
$$

we obtain

$$
D+0.5 E \leq W .
$$

Therefore, the conditions for four active days-off patterns (4, 6,10 , and 14) are

$$
\begin{gathered}
W=\left\lceil\frac{4 D}{3}\right\rceil \\
D+0.5 E \leq W .
\end{gathered}
$$

If the two conditions in (28a)-(28b) are satisfied, then from (23a) and (23c) the bounds on $x_{4}$ are given by

$$
D-0.5 W \leq x_{4} \leq 0.5 W-0.5 E \text {. }
$$

If the interval between the bounds in (29) contains integer values, then $x_{4}$ is set equal to the maximum integer value in this interval, that is, the upper bound rounded down. Otherwise, $x_{4}$ is set equal to the nearest integer to the interval, that is, the lower bound in (29) rounded up. Feasible assignments to the four days-off patterns $(4,6,10$, and 14) are calculated as follows:

$$
\begin{gathered}
x_{4}=\max \{\lceil D-0.5 W\rceil,\lfloor 0.5 W-0.5 E\rfloor\} \\
x_{6}=W-E-x_{4} \\
x_{10}=\left\lceil\frac{E}{2}\right\rceil \\
x_{14}=E-x_{10}
\end{gathered}
$$

where $\lfloor c\rfloor=c$ rounded down to the nearest integer.

The above solution has to be checked to verify that weekend-off frequency constraints are satisfied. Noting that patterns 10 and 14 belong to $J_{1}$ while patterns 4 and 6 belong to $\mathrm{J}_{2}$ and using (8), the actual proportion of weekend-containing days-off breaks is given by

$$
\rho=\frac{0.5\left(x_{10}+x_{14}\right)+\left(x_{4}+x_{6}\right)}{W} .
$$

If $W=\lceil 4 D / 3\rceil$ is approximated by $4 D / 3$ in (28b), we obtain $E \leq 2 D / 3$. Since (30d) gives $x_{10}+x_{14}=E$ and since $E \leq 2 D / 3$ and $W \geq 4 D / 3$, then $\rho$ is approximated as shown below. Since this proportion is greater than 0.5 , weekend-off frequency constraints are satisfied:

$$
\rho \approx \frac{0.5(2 D / 3)+(2 D / 3)}{4 D / 3}=0.75 .
$$

Next, the four-pattern solution needs to be checked against work stretch constraints. The presence of days-off patterns 10 and 14 guarantees the satisfaction of work stretch constraints by assuring a feasible rotation sequence. As noted before, pattern 10 may precede any pattern, while pattern 14 can either follow or precede any pattern. Many feasible cyclic rotation sequences exist for the four patterns that satisfy work stretch constraints. The only restriction is to avoid following pattern 4 immediately by pattern 10 . For example, the simple sequence (4-6-10-14) is feasible in terms of satisfying work stretch constraints.

5.2. Seven Active Patterns. If any of the conditions in (28a)(28b) are not satisfied, then assignment to four days-off patterns is not feasible. Under certain conditions, assignment to seven days-off patterns $(2,4,6,8,10,12$, and 14) is feasible for both arguments of $W$ specified in (20). Assuming $\left(x_{4}=\right.$ $\left.x_{6}=x_{10}=x_{14}\right)$ and $\left(x_{2}=x_{8}=x_{12}\right)$ and ignoring 
redundant constraints, the daily labor demand constraints are represented as follows:

$$
\begin{aligned}
& 3 x_{4}+2 x_{2} \geq D \\
& 2 x_{4}+3 x_{2} \geq E
\end{aligned}
$$

where

$$
4 x_{4}+3 x_{2}=W .
$$

Combining (32a) with (32c) gives

$$
x_{4} \geq 3 D-2 W \text {. }
$$

Combining (32b) with (32c) gives

$$
x_{4} \leq \frac{(W-E)}{2} .
$$

Setting the lower bound in (33a) less than or equal to the upper bound in (33b) gives the following condition for seven active days-off patterns:

$$
1.2 D+0.2 E \leq W
$$

If the above condition is satisfied, then the values $x_{4}=x_{6}=$ $x_{10}=x_{14}$ are set equal to the maximum integer value in or nearest to the interval defined by (33a)-(33b). The remainder of $W$ is assigned to patterns 2,8 , and 12 , giving priority to pattern 12 because it has a full weekend off. Assignments to all seven patterns are calculated as follows:

$$
\begin{gathered}
x_{4}=x_{6}=x_{10}=x_{14}=\max \{\lceil 3 D-2 W\rceil,\lfloor 0.5 W-0.5 E\rfloor\} \\
x_{12}=\left\lceil\frac{\left(W-4 x_{4}\right)}{3}\right\rceil \\
x_{2}=\left\lceil\frac{\left(W-4 x_{4}-x_{12}\right)}{2}\right\rceil \\
x_{8}=W-4 x_{4}-x_{12}-x_{2}
\end{gathered}
$$

Using (8), it can be shown that the percentage of weekendcontaining days off is approximately $75 \%(\rho \approx 0.75)$. There are many feasible cyclic rotation sequences for the seven days-off patterns. For example, the rotation sequence (2-4-6-8-10-1214) guarantees that work stretch limits are not exceeded.

5.3. Eight Active Patterns. If $W=\lceil 4 D / 3\rceil$ and assignment to four or seven days-off patterns is not feasible, then it may be possible to allocate the $W$ employees among eight patterns $(3,4,6,7,10,11,13,14)$. Assuming $\left(x_{4}=x_{6}=x_{10}=x_{14}\right)$ and $\left(x_{3}=x_{7}=x_{11}=x_{13}\right)$ and ignoring redundant constraints, the daily labor demand constraints can be expressed as

$$
\begin{aligned}
& 3 x_{4}+3 x_{3} \geq D \\
& 2 x_{4}+3 x_{3} \geq E \\
& 3 x_{4}+2 x_{3} \geq E,
\end{aligned}
$$

where

$$
4 x_{4}+4 x_{3}=W \text {. }
$$

Combining (36c) with (36d) gives

$$
x_{4} \geq E-0.5 W \text {. }
$$

Combining the sum of (36a) and (36b) with (36d) gives

$$
x_{4} \leq 1.5 W-D-E \text {. }
$$

The first condition for a feasible solution with eight days-off patterns $(3,4,6,7,10,11,13$, and 14$)$ is

$$
W=\left\lceil\frac{4 D}{3}\right\rceil .
$$

Setting the lower bound in (37a) less than or equal to the upper bound in (37b) leads to the second condition for eightpattern assignment:

$$
0.5 D+E \leq W
$$

If (38a)-(38b) is satisfied, the values $x_{4}=x_{6}=x_{10}=x_{14}$ are set equal to the maximum integer value in or nearest to the interval defined by (37a)-(37b). In assigning patterns $(3,7,11$, 13 ), priority is given to patterns 11 and 13 because they contain full weekends off. Assignments to the eight days-off patterns are calculated as follows:

$$
\begin{gathered}
x_{4}=x_{6}=x_{10}=x_{14}=\max \{\lceil E-0.5 W\rceil,\lfloor 1.5 W-D-E\rfloor\} \\
x_{11}=\left\lceil\frac{\left(W-4 x_{4}\right)}{4}\right\rceil \\
x_{13}=\left\lceil\frac{\left(W-4 x_{4}-x_{11}\right)}{3}\right\rceil \\
x_{3}=\left\lceil\frac{\left(W-4 x_{4}-x_{11}-x_{13}\right)}{2}\right\rceil \\
x_{7}=W-4 x_{4}-x_{11}-x_{13}-x_{3} .
\end{gathered}
$$

The proportion of weekend days off can be shown to be approximately equal to 0.75 . To satisfy work stretch constraints, there are many feasible cyclic rotation sequences, such as the sequence (3-4-6-7-10-11-13-14).

5.4. Ten Active Patterns. Assignment to 10 days-off patterns $(2,3,4,6,7,9,10,11,13,14)$ is applicable when $W=\lceil D+0.4 E\rceil$ and a seven-pattern solution is not feasible. Assuming $\left(x_{4}=\right.$ $\left.x_{6}=x_{10}=x_{14}\right),\left(x_{3}=x_{7}=x_{11}=x_{13}\right)$, and $\left(x_{2}=x_{9}\right)$, the active daily labor demand constraints can be expressed as follows:

$$
\begin{aligned}
& 3 x_{4}+3 x_{3}+x_{2} \geq D \\
& 2 x_{4}+3 x_{3}+2 x_{2} \geq E \\
& 3 x_{4}+2 x_{3}+2 x_{2} \geq E
\end{aligned}
$$


where

$$
4 x_{4}+4 x_{3}+2 x_{2}=W
$$

The linear combination $2 \times(40 a)+(40 c)-2 \times(40 d)$ gives

$$
x_{4} \geq 2 D+E-2 W \text {. }
$$

The linear combination $-(40 a)-(40 b)+1.5 \times(40 d)$ gives

$$
x_{4} \leq 1.5 W-D-E \text {. }
$$

The first condition for a 10-pattern solution is

$$
W=\lceil D+0.4 E\rceil \text {. }
$$

Setting the lower bound in (41a) less than or equal to the upper bound in (41b) gives the second condition:

$$
\frac{(6 D+4 E)}{7} \leq W
$$

After fixing $\left(x_{4}=x_{6}=x_{10}=x_{14}\right)$ as the maximum integer value inside or nearest to the interval defined by (41a)-(41b), the bounds on $\mu_{3}$, the average value of $\left(x_{3}=x_{7}=x_{11}=x_{13}\right)$ are found in terms of the $x_{4}$ value. First, the labor constraints system (40a)-(40d) is written as follows:

$$
\begin{gathered}
3 \mu_{3}+x_{2} \geq D-3 x_{4} \\
3 \mu_{3}+2 x_{2} \geq E-2 x_{4} \\
2 \mu_{3}+2 x_{2} \geq E-3 x_{4}
\end{gathered}
$$

where

$$
4 \mu_{3}+2 x_{2}=W-4 x_{4}
$$

The linear combination $2 \times(43 \mathrm{a})-(43 \mathrm{~d})$ gives

$$
\mu_{3} \geq D-0.5 W-x_{4}
$$

Subtracting (43d) - (43b) gives

$$
\mu_{3} \leq W-E-2 x_{4} .
$$

Subtracting $(43 d)-(43 c)$ gives

$$
\mu_{3} \leq \frac{\left(W-E-x_{4}\right)}{2} .
$$

It can be shown that the upper bound in (44c) dominates the one in (44b) because if $\left[\left(W-E-x_{4}\right) / 2 \leq W-E-2 x_{4}\right]$, then $\left(W-E-x_{4} \leq 2 W-2 E-4 x_{4}\right)$, or $\left(E+3 x_{4} \leq W\right)$. Since (41a) specifies $\left(x_{4} \geq 2 D+E-2 W\right)$, then $[E+3(2 D+E-2 W) \leq W]$, or $(6 D+4 E \leq 7 W)$, which is always true for 10-pattern solutions according to condition (42b).

The limits on $\mu_{3}$ in (44a) and (44c) are bounds on the average value of the variables $\left(x_{3}, x_{7}, x_{11}, x_{13}\right)$, not necessarily bounds on the individual value of each variable. If the interval between the bounds in (44a) and (44c) contains integer values, then $\left(x_{3}=x_{7}=x_{11}=x_{13}\right)$ are set equal to the maximum integer value of $\mu_{3}$ in this interval. Otherwise, if the interval contains only one point, specifying a fractional value for $\mu_{3}$, then $\left(x_{3}, x_{7}, x_{11}, x_{13}\right)$ will take different values. Giving priority to patterns 11 and 13 to maximize weekends off, assignments to the 10 days-off patterns are calculated as follows:

$$
\begin{gathered}
x_{4}=x_{6}=x_{10}=x_{14} \\
=\max \{\lceil 2 D+E-2 W\rceil,\lfloor 1.5 W-D-E\rfloor\} \\
\mu_{3}=\max \left\{\left\lceil D-0.5 W-x_{4}\right\rceil,\left\lfloor\frac{\left(W-E-x_{4}\right)}{2}\right]\right\} \\
x_{3}=\left\lceil\frac{4 \mu_{3}}{4}\right\rceil \\
x_{13}=\left\lceil\frac{\left(4 \mu_{3}-x_{3}\right)}{3}\right\rceil \\
x_{11}=\left\lceil\frac{\left(4 \mu_{3}-x_{3}-x_{13}\right)}{2}\right\rceil \\
x_{7}=4 \mu_{3}-x_{3}-x_{11}-x_{13} \\
x_{9}=\left\lceil\frac{\left(W-4 x_{4}-4 \mu_{3}\right)}{2}\right\rceil \\
x_{2}=W-4 x_{4}-4 \mu_{3}-x_{9} .
\end{gathered}
$$

The proportion of weekend days off for the 10-pattern schedule can be shown to be roughly 0.75 , assuring weekend off constraints are satisfied. Work stretch constraints are easily satisfied by many rotation sequences, including the sequence (2-3-4-6-7-9-10-11-13-14).

5.5. Eleven Active Patterns. This solution is applicable when $W=\lceil D+0.4 E\rceil$ and assignment to 7 or 10 patterns is not feasible. In this case, employees are assigned to 11 days-off patterns $\left(1,3,4,5,6,7,9,10,11,13\right.$, and 14). Assuming $\left(x_{4}=\right.$ $\left.x_{6}=x_{10}=x_{14}\right),\left(x_{3}=x_{7}=x_{11}=x_{13}\right)$, and $\left(x_{1}=x_{5}=x_{9}\right)$, the daily labor demand constraints can be written as

$$
\begin{aligned}
& 3 x_{4}+3 x_{3}+2 x_{1} \geq D \\
& 2 x_{4}+3 x_{3}+2 x_{1} \geq E \\
& 3 x_{4}+2 x_{3}+3 x_{1} \geq E,
\end{aligned}
$$

where

$$
4 x_{4}+4 x_{3}+3 x_{1}=W
$$

The linear combination $6 \times(46 a)+(46 c)-5 \times(46 d)$ gives

$$
x_{4} \geq 6 D+E-5 W \text {. }
$$

The linear combination $-6 \times(46 b)-(46 c)+5 \times(46 d)$ gives

$$
x_{4} \leq W-1.4 E \text {. }
$$

The first condition for a feasible 11-pattern solution is

$$
W=\lceil D+0.4 E\rceil \text {. }
$$


TABLE 2: Summary of results for the five cases of days-off assignments.

\begin{tabular}{llllll}
\hline Number of patterns & $W=\lceil$ term $\rceil$ & Condition: term $\leq W$ & Active days-off patterns & Solution system \\
\hline 4 & $4 D / 3$ & $D+0.5 E$ & $4,6,10,14$ & & $(22)$ \\
7 & $4 D / 3$, or $D+0.4 E$ & $1.2 D+0.2 E$ & $4,6,10,14,2,8,12$ & $(26)$ \\
8 & $4 D / 3$ & $0.5 D+E$ & $4,6,10,14,3,7,11,13$ & $(30)$ \\
10 & $D+0.4 E$ & $(6 D+4 E) / 7$ & $4,6,10,14,3,7,11,13,2,9$ & $(36)$ \\
11 & $D+0.4 E$ & $D+0.4 E$ & $4,6,10,14,3,7,11,13,1,5,9$ & $(42)$ \\
\hline
\end{tabular}

TABLE 3: Cyclic rotation schedule for the real-life example.

\begin{tabular}{lccccccccccccc}
\hline Employee & Weeks & 1,2 & 3,4 & 5,6 & 7,8 & 9,10 & 11,12 & 13,14 & 15,16 & 17,18 & 19,20 & 21,22 & 23,24 \\
\hline 1 & 3 & $\mathbf{4}$ & $\mathbf{4}$ & $\mathbf{6}$ & $\mathbf{6}$ & 7 & 10 & 10 & $\mathbf{1 1}$ & $\mathbf{1 3}$ & 14 & 14 \\
2 & 14 & 3 & $\mathbf{4}$ & $\mathbf{4}$ & $\mathbf{6}$ & $\mathbf{6}$ & 7 & 10 & 10 & $\mathbf{1 1}$ & $\mathbf{1 3}$ & 14 \\
3 & 14 & 14 & 3 & $\mathbf{4}$ & $\mathbf{4}$ & $\mathbf{6}$ & $\mathbf{6}$ & 7 & 10 & 10 & $\mathbf{1 1}$ & $\mathbf{1 3}$ \\
4 & $\mathbf{1 3}$ & 14 & 14 & 3 & $\mathbf{4}$ & $\mathbf{4}$ & $\mathbf{6}$ & $\mathbf{6}$ & 7 & 10 & 10 & $\mathbf{1 1}$ \\
5 & $\mathbf{1 1}$ & $\mathbf{1 3}$ & 14 & 14 & 3 & $\mathbf{4}$ & $\mathbf{4}$ & $\mathbf{6}$ & $\mathbf{6}$ & 7 & 10 & 10 \\
6 & 10 & $\mathbf{1 1}$ & $\mathbf{1 3}$ & 14 & 14 & 3 & $\mathbf{4}$ & $\mathbf{4}$ & $\mathbf{6}$ & $\mathbf{6}$ & 7 & 10 \\
7 & 10 & 10 & $\mathbf{1 1}$ & $\mathbf{1 3}$ & 14 & 14 & 3 & $\mathbf{4}$ & $\mathbf{4}$ & $\mathbf{6}$ & $\mathbf{6}$ & 7 \\
8 & 7 & 10 & 10 & $\mathbf{1 1}$ & $\mathbf{1 3}$ & 14 & 14 & 3 & $\mathbf{4}$ & $\mathbf{4}$ & $\mathbf{6}$ & $\mathbf{6}$ \\
9 & $\mathbf{6}$ & 7 & 10 & 10 & $\mathbf{1 1}$ & $\mathbf{1 3}$ & 14 & 14 & 3 & $\mathbf{4}$ & $\mathbf{4}$ & $\mathbf{6}$ \\
10 & $\mathbf{6}$ & $\mathbf{6}$ & 7 & 10 & 10 & $\mathbf{1 1}$ & $\mathbf{1 3}$ & 14 & 14 & 3 & $\mathbf{4}$ & $\mathbf{4}$ \\
11 & $\mathbf{4}$ & $\mathbf{6}$ & $\mathbf{6}$ & 7 & 10 & 10 & $\mathbf{1 1}$ & $\mathbf{1 3}$ & 14 & 14 & 3 \\
12 & $\mathbf{4}$ & $\mathbf{4}$ & $\mathbf{6}$ & $\mathbf{6}$ & 7 & 10 & 10 & $\mathbf{1 1}$ & $\mathbf{1 3}$ & 14 & $\mathbf{4}$ \\
\hline
\end{tabular}

Setting the lower bound in (47a) less than or equal to the upper bound in (47b) leads to

$$
D+0.4 E \leq W .
$$

This condition is redundant since $W=\lceil D+0.4 E\rceil$. After fixing the values of $\left(x_{4}=x_{6}=x_{10}=x_{14}\right)$ within the bounds in (47a)-(47b), the bounds on $x_{3}$ can be calculated in terms of $x_{4}$ as follows:

$$
\begin{aligned}
& 3 x_{3}+2 x_{1} \geq D-3 x_{4} \\
& 3 x_{3}+2 x_{1} \geq E-2 x_{4} \\
& 2 x_{3}+3 x_{1} \geq E-3 x_{4}
\end{aligned}
$$

where

$$
4 x_{3}+3 x_{1}=W-4 x_{4} .
$$

Constraint (50a) dominates (50b) because if ( $D-3 x_{4} \geq E-$ $\left.2 x_{4}\right)$, then $\left(D \geq E+x_{4}\right)$, but since (47a) specifies $\left(x_{4} \geq 6 D+\right.$ $E-5 W)$, then $(D \geq E+6 D+E-5 W)$, or $(W \geq D+0.4 E)$, which is always true according to (16).

The linear combination $3 \times(50 \mathrm{a})-2 \times(50 \mathrm{~d})$ gives

$$
x_{3} \geq 3 D-2 W-x_{4} .
$$

Subtracting $(50 \mathrm{~d})-(50 \mathrm{c})$ gives

$$
x_{3} \leq \frac{\left(W-E-x_{4}\right)}{2} .
$$

The values of $\left(x_{3}=x_{7}=x_{11}=x_{13}\right)$ are set equal to the maximum integer value between the bounds in (51a)-(51b).
Giving priority to patterns 5, which has a full weekend off, employees are assigned to the 11 days-off patterns as follows:

$$
\begin{gathered}
x_{4}=x_{6}=x_{10}=x_{14}=\max \{\lceil 6 D+E-5 W\rceil,\lfloor W-1.4 E\rfloor\} \\
x_{3}=x_{7}=x_{11}=x_{13} \\
\left.=\max \left\{\left\lceil 3 D-2 W-x_{4}\right\rceil, \mid \frac{\left(W-E-x_{4}\right)}{2}\right]\right\} \\
x_{5}=\left\lceil\frac{\left(W-4 x_{4}-4 x_{3}\right)}{3}\right] \\
x_{1}=\left\lceil\frac{\left(W-4 x_{4}-4 x_{3}-x_{5}\right)}{2}\right\rceil \\
x_{9}=W-4 x_{4}-4 x_{3}-x_{5}-x_{1} .
\end{gathered}
$$

Applying (8), it can be shown that the 11-pattern schedule satisfies the weekend-off constraints because the proportion of breaks with weekends off is approximately equal to 0.75 . Work stretch constraints are easily satisfied by many rotation sequences, including the sequence (1-2-3-4-5-6-7-9-10-11-1314).

The results obtained in Section 5 are summarized in Table 2 . It is clear that one set of active patterns $(4,6,10$, and 14 ) is used consistently for all cases, while other sets such as patterns $(3,7,11$, and 13$)$ are used to satisfy most labor demands variations. 


\section{Application and Example}

6.1. The Scheduling Process. The optimal solution results, obtained in Sections 4 and 5 and summarized in Table 2, have been incorporated into a simple spreadsheet tool. This userfriendly tool can be easily used to find the optimal schedule directly, without having to run any optimization solver. By simply entering the weekday and weekend daily labor demands $D$ and $E$, respectively, the spreadsheet immediately shows the workforce size, the number of days-off patterns, and the number of employees assigned to each pattern. Because most remote area employees are technicians with low IT skills, the spreadsheet tool avoids the use of specialized optimization software. The spreadsheet is easy to use and has been well accepted by the concerned employees and their supervisors. To illustrate the calculations used to find the optimum $(10,14)$ schedule, a real-life example from one of the locations areas is solved below.

6.2. A Real-Life Example. For a remote work site, daily labor demand is nine employees during regular weekdays and seven employees during weekends. The days-off schedule is determined as follows.

Given

$$
D=9, \quad E=7,
$$

using (20),

$$
W=\left\lceil\max \left\{\frac{4 \times 9}{3}, 9+0.4 \times 7\right\}\right\rceil=\lceil\max \{12,11.8\}\rceil=12 .
$$

Checking 4-pattern condition (28b): $9+0.5(7)=$ $12.5>W$ (infeasible);

checking 7-pattern condition (34): 1.2(9) + 0.2(7) = $12.2>W$ (infeasible);

checking 8-pattern condition (38b): $0.5(9)+7=$ $11.5 \leq W$ (feasible);

calculating the bounds on $x_{4}$ for 8 patterns:

$$
\begin{aligned}
& \text { using (37a): } x_{4} \geq 7-0.5(12)=1, \\
& \text { using (37b): } x_{4} \leq 1.5 \times 12-9-7=2,
\end{aligned}
$$

using the equation system (39a)-(39e),

$$
\begin{gathered}
x_{4}=x_{6}=x_{10}=x_{14}=2 \\
x_{3}=x_{7}=x_{11}=x_{13}=\frac{(12-4 \times 2)}{4}=1,
\end{gathered}
$$

using (8),

$$
\begin{aligned}
\rho & =\frac{0.5\left(x_{3}+x_{7}+x_{10}+x_{14}\right)+\left(x_{4}+x_{6}+x_{11}+x_{13}\right)}{W} \\
& =\frac{0.5(1+1+2+2)+(2+2+1+1)}{12}=0.75 .
\end{aligned}
$$

Following the cyclic sequence (3-4-6-7-10-11-13-14) and using a cycle of 24 weeks, the 12 employees are rotated on the 8 daysoff patterns as shown in Table 3 . The numbers inside each cell indicate the assigned days-off patterns for the given employee in the particular week. Shaded cells indicate assignment to a $J_{2}$ days-off pattern, that is, two weekend days off.

\section{Conclusions and Extensions}

A model and optimum solution have been presented for a real-life employee days-off scheduling problem with unique features. An innovative practical approach has been used, in which simple practical solutions are developed to deal with theoretically complex issues. The problem is to assign remote-area employees to the cyclic $(10,14)$ days-off schedule, primarily in order to minimize workforce size. The secondary objective is to reduce the costs of employee air transportation by minimizing the number of active days-off patterns. The constraints include daily labor demands, weekend off frequency requirements, work stretch limitations, and fair workload distribution. This optimization problem has been modeled as a biobjective pure-integer linear programming model with both binary and general integer variables. The distinctive structure of the model and the given real-life values of problem parameters have been utilized to develop a simple solution procedure that eliminates the need to use integer-programming solvers.

The work presented in this paper can be extended in several directions. For example, different labor demands for each day can be considered instead of two fixed demand levels ( $D$ for workdays and $E$ for weekends). Another possibility is to consider the case where weekend labor demands are higher than weekday demands. These two extensions are not applicable to the current real-life problem presented in this paper but might be applicable either in the future or in other real-life scheduling situations. Finally, integrating transportation (flight) scheduling and employee scheduling for multiple work locations presents an interesting and challenging research opportunity.

\section{Conflict of Interests}

The author declares that there is no conflict of interests regarding the publication of this paper.

\section{References}

[1] H. K. Alfares, "Survey, categorization, and comparison of recent tour scheduling literature," Annals of Operations Research, vol. 127, pp. 145-175, 2004.

[2] A. T. Ernst, H. Jiang, M. Krishnamoorthy, and D. Sier, "Staff scheduling and rostering: a review of applications, methods and models," European Journal of Operational Research, vol. 153, no. 1, pp. 3-27, 2004.

[3] J. O. Brunner, "Literature review on personnel scheduling," in Flexible Shift Planning in the Service Industry: The Case of Physicians in Hospitals, vol. 640 of Lecture Notes in Economics and Mathematical Systems, pp. 5-12, Springer, Berlin, Germany, 2010. 
[4] P. Brucker, R. Qu, and E. Burke, "Personnel scheduling: models and complexity," European Journal of Operational Research, vol. 210, no. 3, pp. 467-473, 2011.

[5] F. Jarray, "Solving problems of discrete tomography: application in workforce scheduling," 4OR: A Quarterly Journal of Operations Research, vol. 3, no. 4, pp. 337-340, 2005.

[6] M. C. Costa, F. Jarray, and C. Picouleau, "An acyclic days-off scheduling problem," 4OR, vol. 4, no. 1, pp. 73-85, 2006.

[7] F. Jarray, "A 4-day or a 3-day workweeks scheduling problem with a given workforce size," Asia-Pacific Journal of Operational Research, vol. 26, no. 5, pp. 685-696, 2009.

[8] M. T. Lilly, I. Emovon, S. O. T. Ogaji, and S. D. Probert, "Fourday service-staff work-week in order to complete maintenance operations more effectively in a Nigerian power-generating station," Applied Energy, vol. 84, no. 10, pp. 1044-1055, 2007.

[9] H. K. Alfares, "A simulation approach for stochastic employee days-off scheduling," International Journal of Modelling and Simulation, vol. 27, no. 1, pp. 9-15, 2007.

[10] M. Elshafei and H. K. Alfares, "A dynamic programming algorithm for days-off scheduling with sequence dependent labor costs," Journal of Scheduling, vol. 11, no. 2, pp. 85-93, 2008.

[11] A. Klinkert, "Days-off scheduling in large-scale multi-skill staff rostering: an integer programming solution," in Proceedings of the 7th International Conference on the Practice and Theory of Automated Timetabling (PATAT'08), Montréal, Canada, August 2008.

[12] B. Maenhout and M. Vanhoucke, "Days on and days off scheduling of pilots under a variable workload," in Airline Industry: Strategies, Operations and Safety, C. R. Walsh, Ed., pp. 193-212, 2011.

[13] J. Kyngäs and K. Nurmi, "Days-off scheduling for a bus transportation company," International Journal of Innovative Computing and Applications, vol. 3, no. 1, pp. 42-49, 2011.

[14] R. Lusby, A. Dohn, T. M. Range, and J. Larsen, "A column generation-based heuristic for rostering with work patterns," Journal of the Operational Research Society, vol. 63, no. 2, pp. 261-277, 2012.

[15] A. Abdoul Soukour, L. Devendeville, C. Lucet, and A. Moukrim, "A Memetic Algorithm for staff scheduling problem in airport security service," Expert Systems with Applications, vol. 40, no. 18, pp. 7504-7512, 2013.

[16] A. Rong, "Monthly tour scheduling models with mixed skills considering weekend off requirements," Computers and Industrial Engineering, vol. 59, no. 2, pp. 334-343, 2010.

[17] G. M. Campbell, "A two-stage stochastic program for scheduling and allocating cross-trained workers," Journal of the Operational Research Society, vol. 62, no. 6, pp. 1038-1047, 2011.

[18] H. K. Alfares, "A dual-based combinatorial algorithm for solving cyclic optimization problems," Recent Patents on Computer Science, vol. 5, no. 3, pp. 188-196, 2012. 

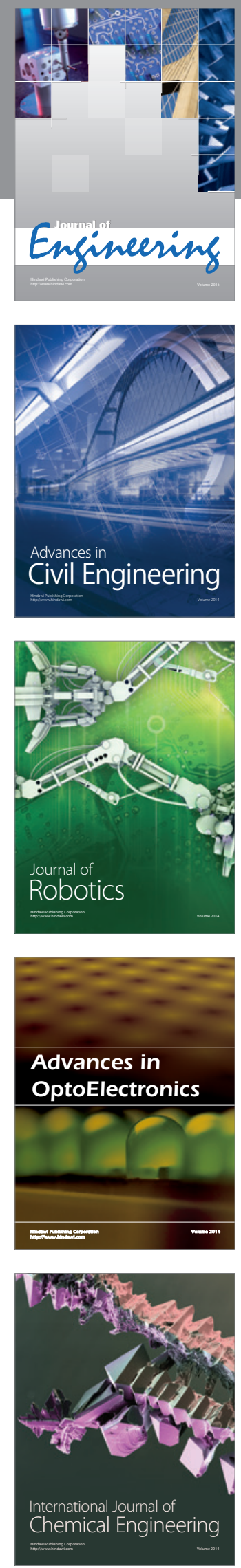

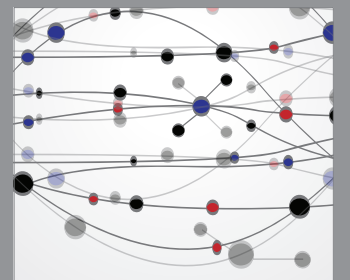

The Scientific World Journal
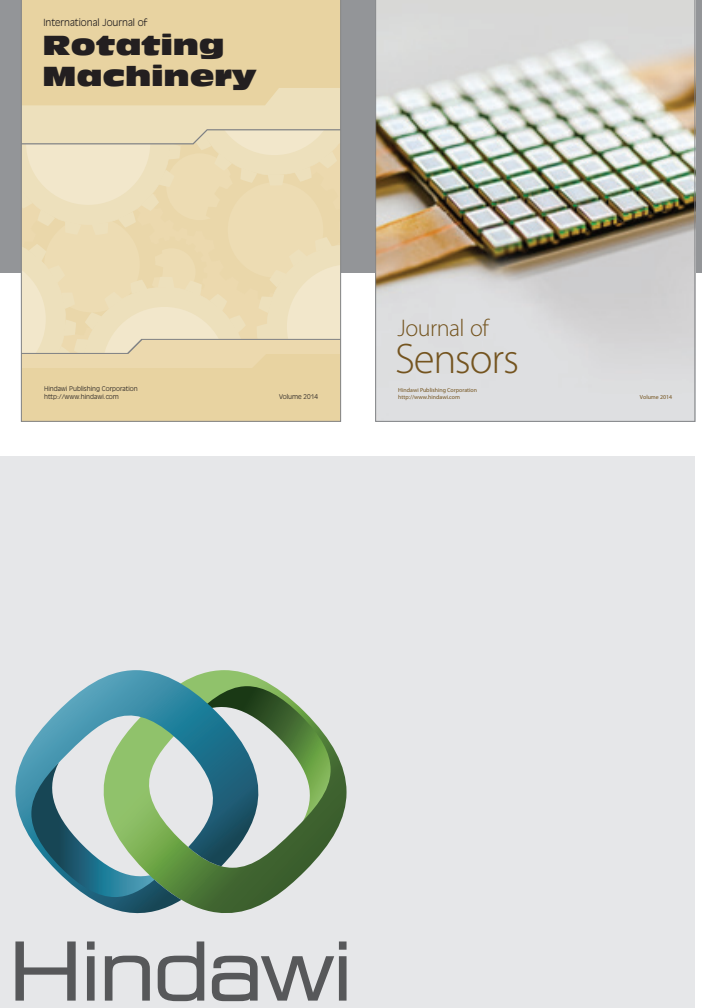

Submit your manuscripts at http://www.hindawi.com
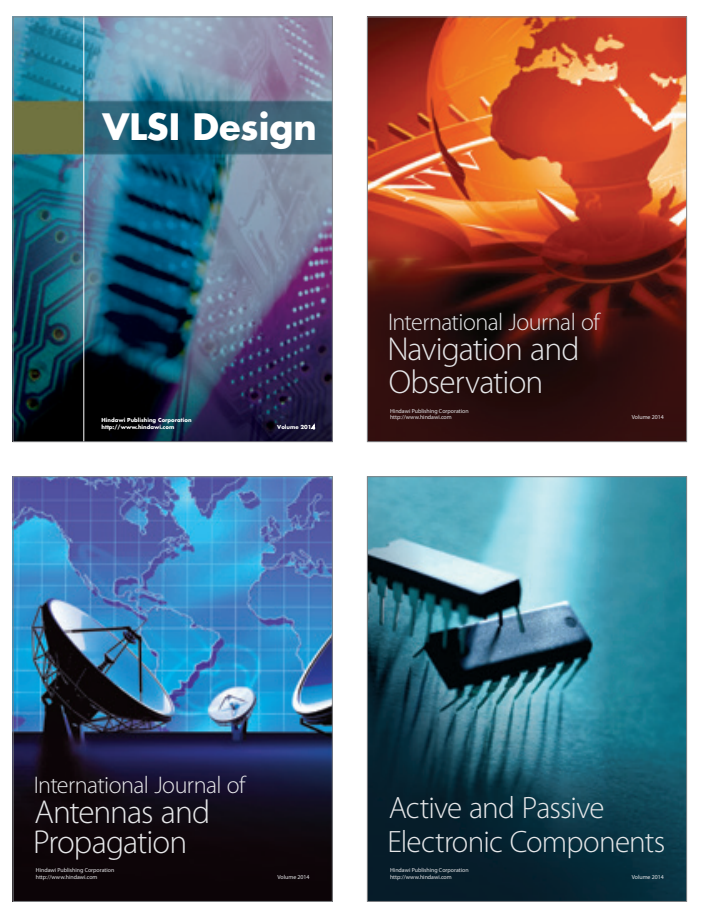
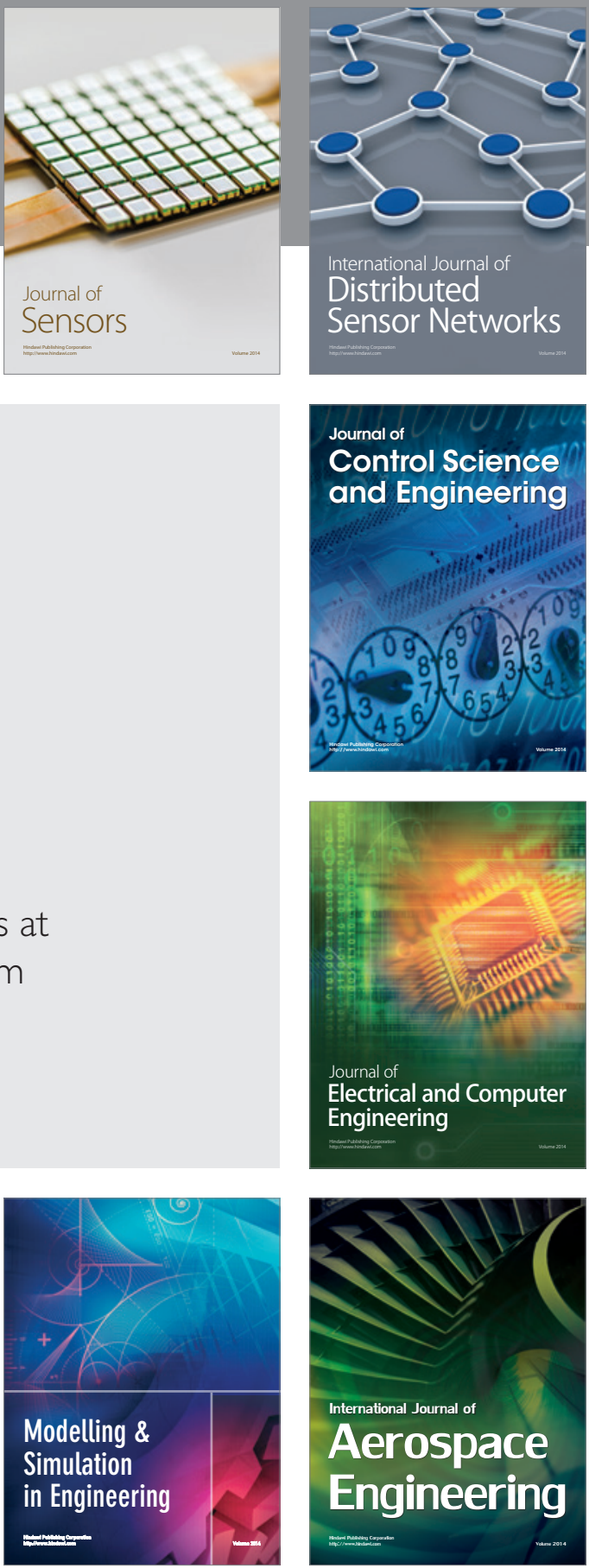

Journal of

Control Science

and Engineering
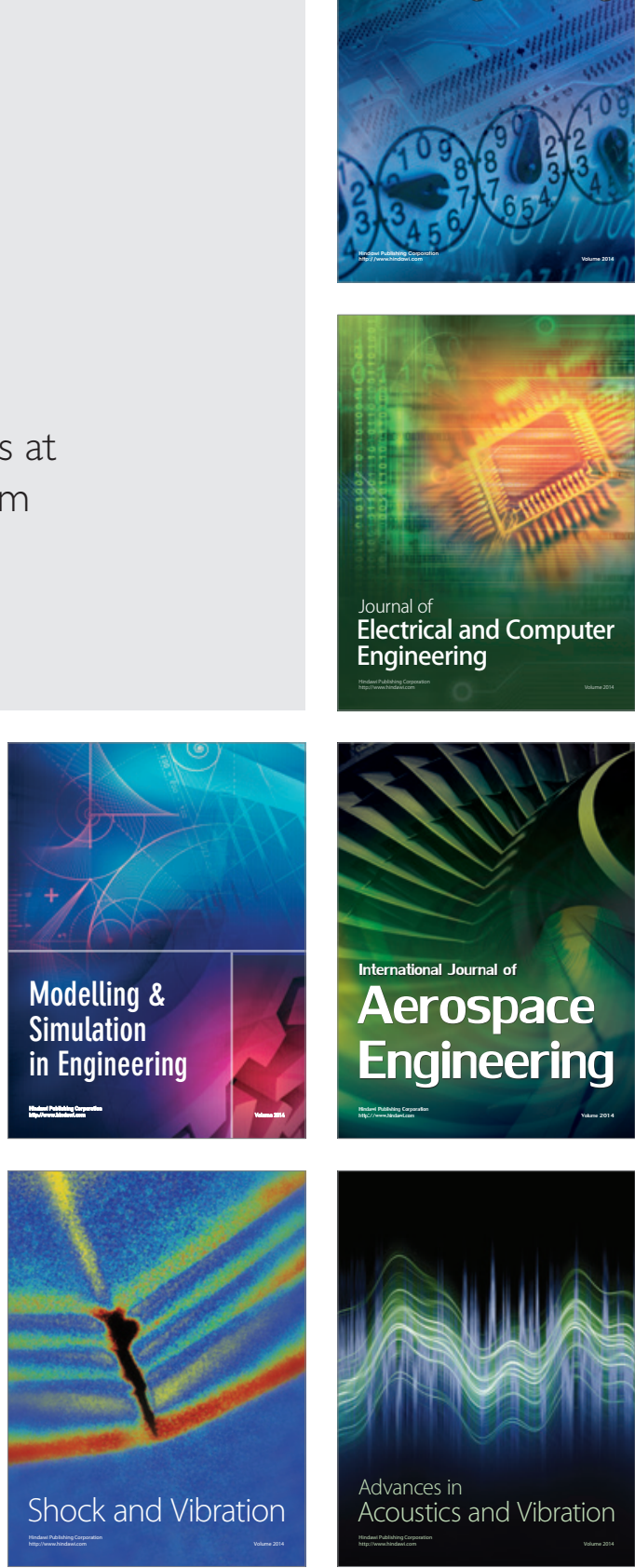